\title{
Analytical solution of Klein Gordon equation Trigonometric Pöschl-Teller potential using asymptotic iteration method
}

\author{
Dewanta Arya Nugraha ${ }^{1}$, A. Suparmi ${ }^{2}$, C. Cari $^{2}$ \\ ${ }^{1,2}$ Physics Department, Graduate Program, Sebelas Maret University, INDONESIA \\ Jl. Ir. Sutami 36A Kentingan Jebres Surakarta 57126 \\ ${ }^{1}$ Email : dewanta.an@gmail.com
}

Received 18 October 2016, Revised 16 November 2016, Accepted 20 December 2016

\begin{abstract}
Radial part of Klein Gordon equation for trigonometric PöschlTeller potential was obtained within framework of a centrifugal term approximation. The relativistic energy spectrum and wave functions was obtain by using asymptotic iteration method. The value of relativistic energy was calculated numerically using Matlab 2013. The results showed that the relativistic energy is increasing due to the increase of potential constant and quantum number.
\end{abstract}

Keywords: Klein Gordon; Trigonometric Pöschl-Teller Potential; Asymptotic Iteration Method (AIM).

\section{Introduction}

Solution of Klein Gordon, Dirac, and Duffin-Kemmer-Petiau equation with various potential is very important in nuclear physics and high energy physics. This equation is frequently used to describe the dynamics particle in relativistic quantum physics (Ikhdair \& Sever, 2008). There are many research focus on the solution of equation with physical potential. Klein-Gordon equation allows us to study spin-zero particles (Momtazi, Rajabi, \& Yazarloo, 2014). Klein-Gordon Solutions have been studied by using various techniques, they are Standard Method (Xu, He, \& Jia, 2010), Nikiforov Uvarov (NU) Method (Ikhdair, 2011), Supersymmetric Quantum Mechanics (SUSY)(Setare \& Nazari, 2009), Factorization Method (Wei, Liu, \& Chen, 2009), Asymptotic Iteration Method (AIM) (Barakat, 2006)(Das, 2014) and many others.

In this research, the relativistic solution of spin-less particle with Trigonometric Pöschl-Teller Potential is studied. The method used is asymptotic iteration method. Asymptotic Iteration Methods (AIM) is an alternative method which has accuracy and high efficiency to determine eigen energies and eigenfunctions for analytically. Asymptotic Iteration Methods is also giving solution for exactly solvable problem. (Suparmi, Cari, Deta, Husein, \& Yuliani, 2014). 


\section{Klein Gordon Equation}

The equation of time independent KG with vector $V(r)$ and scalar $S(r)$ potentials, where spinless particle is describing with $r=|\mathbf{r}|$ which can be written as (Bayrak \& Boztosun, 2006; Qiang \& Dong, 2007)

$$
\left\{\frac{d^{2}}{d r^{2}}+\frac{1}{\hbar^{2} c^{2}}\left[(E-V(r))^{2}-\left(M c^{2}+S(r)\right)^{2}\right]\right\} \psi(\mathbf{x})=0,
$$

and

$$
\psi(\mathrm{x})=R_{\ell}(r) Y_{j, m}^{\ell}(\theta, \varphi)
$$

$E$ and $M$ express the Klein Gordon energy and mass. And then $\mathbf{x}$ is vector dimensional of position. The radial part wave function $R_{\ell}(r)$ is define as

$$
R_{\ell}(r)=\frac{F(r)}{r}
$$

For limit non-trivial nonrelativistic result for case $S=V$, we get a potential function $2 \mathrm{~V}$ and not $V$. in nonrelativistic limit, the interaction of potential is become $\mathrm{V}$ and not $2 \mathrm{~V}$. That is why equation (1) can be reduced into radial part of 3-Dimensional Klein Gordon equation. (in the relativistic atomic units $\hbar=c=1$ )(Alhaidari, Bahlouli, \& AlHasan, 2006),

$$
\frac{d^{2}}{d r^{2}} \psi(\mathrm{x})+\left[E^{2}-M^{2}-(E+M) V(r)\right] \psi(\mathrm{x})=0
$$

\section{Asymptotic Iteration Method (AIM)}

Asymptotic Iteration Method (AIM) is one of alternative method which has high efficiency and accuracy to determine eigenenergy and eigenfunction. Asymptotic Iteration Method is also giving exactly solvable problem solution (Pramono, Suparmi, \& Cari, 2016).

$$
y_{n}{ }^{\prime \prime}(x)=\lambda_{0}(x) y_{n}{ }^{\prime}(x)+s_{0}(x) y_{n}(x)
$$

with $\lambda_{0}(x) \neq 0$ and prime denoted derivation respect to $x . n$ Parameter is the radial quantum number. Variable $\lambda_{0}(x)$ and $s_{0}(x)$ are differentiable. Equation (4) is differentiated to $x$ to get the solution, and we get

$$
y_{n}{ }^{\prime \prime}(x)=\lambda_{1}(x) y_{n}{ }^{\prime}(x)+s_{1}(x) y_{n}(x)
$$

where

$$
\begin{gathered}
\lambda_{1}(x)=\lambda_{0}{ }^{\prime}(x)+s_{0}(x)+\lambda_{0}^{2}(\mathrm{x}) \\
s_{1}(x)=s_{0}{ }^{\prime}(x)+s_{0}(x) \lambda_{0}(\mathrm{x})
\end{gathered}
$$

In this case $\lambda_{0}(x) \neq 0$ and $s_{0}(x)$ is function of $C_{\infty}$ (coefficient of differential equation). Asymptotic Iteration Method can be applied exactly for different problem if the wave function is known and fulfill the boundaries of condition zero (0) and infinite $(\infty)$.

Equation (5) can be simply iterated until $(k+1)$ and $(k+2), k=1,2,3, \ldots$ then the result become 


$$
\begin{aligned}
& y_{n}{ }^{k+1}(x)=\lambda_{k-1}(x) y_{n}{ }^{\prime}(x)+s_{k-1}(x) y_{n}(x) \\
& y_{n}{ }^{k+2}(x)=\lambda_{k}(x) y_{n}^{\prime}(x)+s_{k}(x) y_{n}(x)
\end{aligned}
$$

where

$$
\begin{gathered}
\lambda_{k}(x)=\lambda_{k-1}{ }^{\prime}(x)+s_{k-1}(x)+\lambda_{0}(x) \lambda_{k-1}(x) \\
s_{k}(x)=s_{k-1}{ }^{\prime}(x)+s_{0}(x) \lambda_{k-1}(x)
\end{gathered}
$$

Which call as recurrence relation. Eigen value can be found using the following equation

$$
\Delta_{k}(x)=\lambda_{k}(x) s_{k-1}(x)-\lambda_{k-1}(x) s_{k}(x)=0
$$

With $k=1,2,3, \ldots$ is iteration number and $n$ is the quantum number for radial part. Equation (5) is the linear homogenous equation in the second orde which can be solved by comparing it with the second order of linear equation as follows (Sari, et al., 2015)

$$
y^{\prime \prime}(x)=2\left(\frac{a x^{N+1}}{1-b x^{N+2}}-\frac{t+1}{x}\right) y^{\prime}(x)-\frac{w x^{N}}{1-b x^{N+2}} y(x)
$$

where

$$
\sigma=\frac{2 t+N+3}{N+2}, \quad \rho=\frac{(2 t+1) b+2 a}{(N+2) b}
$$

The wave function of equation (4) is determined using the follows equation

$$
y_{n}(x)=(-1)^{n} C_{2}(\mathrm{~N}+2)^{n}(\sigma)_{n 2} F_{1}\left(-n, \rho+n ; \sigma ; b x^{N+2}\right)
$$

\section{Result}

The Pöschl Teller Potential is define as (Xu- Yang Liu, 2010)

$$
V(\mathrm{r})=\left(\frac{\kappa(\kappa-1)}{\sin ^{2} \alpha r}+\frac{\eta(\eta-1)}{\cos ^{2} \alpha r}\right)
$$

Equation (5) is substitute with equation $(2-4,19)$, and the result is

$$
\left\{\frac{d^{2}}{d r^{2}}+E^{2}-M^{2}-(E+M) \times\left(\frac{\kappa(\kappa-1)}{\sin ^{2} \alpha r}+\frac{\eta(\eta-1)}{\cos ^{2} \alpha r}\right)-\frac{l(l+1)}{r^{2}}\right\} F(r)=0
$$

By using the centrifugal term approximation for $\alpha r<<1$, then $\frac{1}{r^{2}} \cong \frac{\alpha^{2}}{\sin ^{2} \alpha r}$ is substitute in equation (18), we get

$$
\left[\frac{d^{2}}{d r^{2}}-\frac{\alpha^{2}}{\sin ^{2} \alpha r}\left\{\frac{\kappa(\kappa-1)(E+M)}{\alpha^{2}}-l(l+1)\right\}+\frac{\eta(\eta-1)}{\cos ^{2} \alpha r}(E+M)+\left(E^{2}-M^{2}\right)\right] F(r)=0
$$

let

$$
\begin{gathered}
A=\frac{\kappa(\kappa-1)(E+M)}{\alpha^{2}}-l(l+1) \\
B=\frac{\eta(\eta-1)(E+M)}{\alpha^{2}} \\
E_{s}=\frac{\left(E^{2}-M^{2}\right)}{\alpha^{2}}
\end{gathered}
$$


Equation (19) become

$$
\frac{d^{2} F(r)}{d r^{2}}-\frac{A \alpha^{2}}{\sin ^{2} \alpha r} F(r)-\frac{B \alpha^{2}}{\cos ^{2} \alpha r} F(r)+E_{s} \alpha^{2} F(r)=0
$$

Equation (23) can be solve by letting $\cos ^{2} \alpha r=z$, then the result is

$$
z(1-z) \frac{\partial^{2} F(r)}{\partial z^{2}}+\left(\frac{1}{2}-z\right) \frac{\partial F(r)}{\partial z}+\frac{A}{4(1-z)} F(r)+\frac{B}{4 z} F(r)-\frac{E_{s}}{4} F(r)=0
$$

Equation (24) is hypergeometric differential equation which have to differentiate to second orde differential equation in AIM form. Let

$$
F(z)=z^{\delta}(1-z)^{\gamma} f_{n_{r}}(z)
$$

equation (24) is substitute with equation (25), and the result is

$$
\begin{aligned}
& z(1-z) f^{\prime \prime}{ }_{n_{r}}(z)+\left\{2 \delta-(2 \delta+2 \gamma+1) z+\frac{1}{2}\right\} f^{\prime}{ }_{n_{r}}(z)+ \\
& \left\{\frac{4 \delta(\delta-1)+2 \delta+B}{4 z}+\frac{4 \gamma(\gamma-1)+2 \gamma+A}{4(1-z)}-\delta^{2}-2 \delta \gamma-\gamma^{2}-\frac{E_{s}}{4}\right\} f_{n_{r}}(z)=0
\end{aligned}
$$

Let us consider

$$
\begin{aligned}
& B=-4 \delta^{2}+2 \delta \\
& A=-4 \gamma^{2}+2 \gamma
\end{aligned}
$$

Equation (27) is inserted into equation (26), we get

$$
f^{\prime \prime} n_{r}(z)=\frac{(2 \delta+2 \gamma+1) z-\left(2 \delta+\frac{1}{2}\right)}{z(1-z)} f^{\prime}{ }_{n_{r}}(z)+\frac{(\delta+\gamma)^{2}+\frac{E_{s}}{4}}{z(1-z)} f_{n_{r}}(z)=0
$$

The AIM type equation (28) is compared with equation (5), thus

$$
\begin{gathered}
\lambda_{0}=\frac{(2 \delta+2 \gamma+1) z-\left(2 \delta+\frac{1}{2}\right)}{z(1-z)} \\
s_{0}(z)=\frac{(\delta+\gamma)^{2}+\frac{E_{s}}{4}}{z(1-z)}
\end{gathered}
$$

From equation $(11-13)$, we get the general eigen value from equation (28) as follows

$$
E_{s n}=-4(\delta+\gamma+n)^{2}
$$

with

$$
\begin{gathered}
E_{s n}=\frac{\left(E_{n}^{2}-M^{2}\right)}{\alpha^{2}} \\
\delta=\frac{1}{4}+\frac{1}{2} \sqrt{\frac{1}{4}-B} \\
\gamma=\frac{1}{4}+\frac{1}{2} \sqrt{\frac{1}{4}-A}
\end{gathered}
$$

where $E_{n}$ in equation (32) is the eigen value and $n$ is the radial quantum number, $n=0,1,2, \ldots$. By inserting equation $(20-21,32-34)$ into equation (31) the relativistic energy equation is determined as 


$$
\left(M^{2}-E^{2}\right)=\alpha^{2} \times\left\{2 n+1+\sqrt{\frac{1}{4}-\frac{\kappa(\kappa-1)(E+M)}{\alpha^{2}}-l(l+1)}+\sqrt{\frac{1}{4}-\frac{\eta(\eta-1)(E+M)}{\alpha^{2}}}\right\}^{2}
$$

$l$ is the quantum orbital number for radial part solution. From equation (35) can be calculated the relativistic energy numerically by using Matlab 2013. The result is shown in Table 1.

Tabel 1. Relativistic Energy $E_{n}(\mathrm{eV})$ with $M=1 \mathrm{eV}$ and $\alpha=l=1$

\begin{tabular}{cccc}
\hline$n$ & $\kappa=\eta=1.5$ & $\kappa=\eta=2$ & $\kappa=\eta=2.5$ \\
\hline 0 & 2.9075683 & 8.5471005 & 15.7490201 \\
1 & 3.7012565 & 9.0380661 & 16.1007231 \\
2 & 4.2900391 & 9.6599034 & 16.6538691 \\
3 & 4.7787731 & 10.2737006 & 17.2828748 \\
4 & 5.2052211 & 10.8529416 & 17.9271330 \\
5 & 5.5882218 & 11.3962139 & 18.5621132 \\
\hline
\end{tabular}

Based on Table 1. The energy value is affected by potential constant of Trigonometric Pöschl-Teller potential. The energy value is increasing due to the increase of the potential constant. The value of relativistic energy is increasing due to the increase of the quantum number.

By comparing equation (6) and (29), then by using equation (16) and inserting it into equation (17) we get

$$
f_{n}(z)=(-1)^{n_{r}} C_{2}(1)^{n}(2 \delta+1 / 2)_{n} \times{ }_{2} F_{1}(-n, 2 \delta+2 \gamma+1+n, 2 \delta+1 / 2, z)
$$

By inserting equation (37) to $F_{n_{r}}(z)=(z)^{\delta}(1-z)^{\gamma} f_{n_{r}}(z)$, we get

$$
F_{n}(z)=(z)^{\delta}(1-z)^{\gamma} f_{n_{r}}(z)(-1)^{n_{r}} \times C^{\prime}(1)^{n_{r}}(2 \delta+1 / 2)_{n_{r}} \times{ }_{2} F_{1}\left(-n_{r}, 2 \delta+2 \gamma+1+n_{r}, 2 \delta+1 / 2, z\right)
$$

where $C^{\prime}$ is normalization constant and ${ }_{2} F_{1}(a, b, c, z)$ is the hypergeometric function. Table 2.is presented the unnormalized wave function for the radial part in any states solved from equation (38).

Table 2. Wave function for $n=0,1,2$

\begin{tabular}{ll}
\hline$n$ & $F_{n_{r}}(r)$ \\
\hline 0 & $F_{0}(r)=\left(\cos ^{2} \alpha r\right)^{\delta}\left(\sin ^{2} \alpha r\right)^{\gamma} C^{\prime}$ \\
1 & $F_{1}(r)=-\left(\cos ^{2} \alpha r\right)^{\delta}\left(\sin ^{2} \alpha r\right)^{\gamma} C^{\prime}(2 \delta+1 / 2)\left\{1-\frac{(2 \delta+2 \gamma+2)}{\left(2 \delta+\frac{1}{2}\right)}\left(\cos ^{2} \alpha r\right)\right\}$
\end{tabular}




$$
\begin{aligned}
& F_{2}(r)=\left(\cos ^{2} \alpha r\right)^{\delta}\left(\sin ^{2} \alpha r\right)^{\gamma} C^{\prime}(2 \delta+1 / 2)(2 \delta+3 / 2) \times \\
& \left\{1-\frac{2(2 \delta+2 \gamma+3)}{\left(2 \delta+\frac{1}{2}\right)}\left(\cos ^{2} \alpha r\right)+\frac{(2 \delta+2 \gamma+3)(2 \delta+2 \gamma+4)}{\left(2 \delta+\frac{1}{2}\right)\left(2 \delta+\frac{3}{2}\right)}\left(\cos ^{2} \alpha r\right)^{2}\right\}
\end{aligned}
$$

\section{Conclusion}

Klein Gordon equation for Trigonometric Pöschl-Teller potential was obtained by using centrifugal term approximation. This equation was solved by using asymptotic iteration method. The relativistic energy spectrum and the wave function were obtained by using asymptotic iteration method. Based on the result, the relativistic energy is increasing due to the increase of potential constant and the quantum number value.

\section{Acknowledgement}

This research was partly supported by Sebelas Maret University Higher Education Project Grant with contract No. 632/UN27.21/LT/2016

\section{References}

Alhaidari, A. D., Bahlouli, H., \& Al-Hasan, A. (2006). Dirac and Klein-Gordon equations with equal scalar and vector potentials. Physics Letters, Section A: General, Atomic and Solid State Physics, 349(1-4), 87-97. https://doi.org/10.1016/j.physleta.2005.09.008

Barakat, T. (2006). The asymptotic iteration method for dirac and klein gordon equations with a linear scalar potential. International Journal of Modern Physics A, 21(19), 4127-4135.

Bayrak, O., \& Boztosun, I. (2006). Arbitrary $\ell$-state solutions of the rotating Morse potential by the asymptotic iteration method. Journal of Physics A: Mathematical and General, 39(22), 6955. https://doi.org/10.1088/0305$4470 / 39 / 22 / 010$

Das, T. (2014). Exact Solutions of the Klein-Gordon Equation for q-Deformed Manning-Rosen Potential via Asymptotic Iteration Method. arXiv:1409.1457v1 [Quant-Ph], 1-11.

Ikhdair, S. M. (2011). Bound states of the Klein-Gordon equation in D-dimensions with some physical scalar and vector exponential-type potentials including orbital centrifugal term. arXiv:1110.0943v1 [Quant-Ph], 1-25. Retrieved from http://arxiv.org/abs/1110.0943

Ikhdair, S. M., \& Sever, R. (2008). Solution of the D-dimensional Klein-Gordon equation with equal scalar and vector ring-shaped pseudoharmonic potential. arXiv:0808.1002v1 [Quant-Ph], $1-14$. https://doi.org/10.1142/S0129183108012923

Momtazi, E., Rajabi, A. A., \& Yazarloo, B. H. (2014). Analytical solution of the Klein Gordon equation under the Coulomb-like scalar. Turkish Journal of Physics, 
38(1), 81-85. https://doi.org/10.3906/fiz-1305-7

Pramono, S., Suparmi, A., \& Cari, C. (2016). Relativistic Energy Analysis of FiveDimensional q -Deformed Radial Rosen-Morse Potential Combined with q Deformed Trigonometric Scarf Noncentral Potential Using Asymptotic Iteration Method. Advances in High Energy Physics, 2016. https://doi.org/10.1155/2016/7910341

Qiang, W. C., \& Dong, S. H. (2007). Arbitrary 1-state solutions of the rotating Morse potential through the exact quantization rule method. Physics Letters, Section A: General, Atomic and Solid State Physics, 363(3), 169-176. https://doi.org/10.1016/j.physleta.2006.10.091

Setare, M. R., \& Nazari, Z. (2009). Solution of Dirac Equations With Five-Parameter Exponent-Type Potential. Acta Physica Polonica B, 40(10), 2809-2824.

Suparmi, A., Cari, C., Deta, U. A., Husein, A. S., \& Yuliani, H. (2014). Exact Solution of Dirac Equation for q-Deformed Trigonometric Scarf potential with qDeformed Trigonometric Tensor Coupling Potential for Spin and Pseudospin Symmetries Using Romanovski Polynomial. Journal of Physics: Conference Series, 539, 12004. https://doi.org/10.1088/1742-6596/539/1/012004

Wei, G. F., Liu, X. Y., \& Chen, W. L. (2009). The relativistic scattering states of the hulthén potential with an improved new approximate scheme to the centrifugal term. International Journal of Theoretical Physics, 48(6), 1649-1658. https://doi.org/10.1007/s10773-009-9937-9

$\mathrm{Xu}, \mathrm{Y} ., \mathrm{He}, \mathrm{S} ., \quad \&$ Jia, C.-S. (2010). Approximate analytical solutions of the KleinGordon equation with the Pöschl-Teller potential including the centrifugal term. Physica Scripta, 81(4), 45001. https://doi.org/10.1088/0031-8949/81/04/045001 